http://dx.doi.org/10.11646/zoosymposia.13.1.9

http://zoobank.org/urn:lsid:zoobank.org:pub:7C0073D8-9BC6-4F38-BF15-48EF6B400E3C

\title{
Calliostomatidae of the northeast Pacific
}

\author{
PAUL M. TUSKES \\ 3808 Sioux Ave., San Diego, California, 92117,USA.E-mail: tuskes@AOL.com
}

\begin{abstract}
Four genera of Calliostomatidae are known from the northeast Pacific, Akoya, Calliostoma, Otukaia, and Xeniostoma. Fifteen holotypes and all species are illustrated and diagnostic information presented. Approximately one third of the species occur most commonly below $100 \mathrm{~m}$, some species have been collected in excess of 1,000 m. Akoya titanium (McLean, 1984) and $A$. bernardi (McLean, 1984) are recognized as synonyms of $A$. platinum (Dall, 1890). Confirmed diet of the species, which are known, includes primarily sessile invertebrates and diatoms.
\end{abstract}

Key Words: Calliostomatidae, Calliostoma, Otukaia, Xeniostoma, Akoya northeast Pacific

\section{Introduction}

Although morphological and dietary differences between calliostomids and Trochidae were previously known (Hickman \& McLean 1990, Perron 1975), it was not until DNA data indicated the group was distinct, that Calliostomatidae Thiele (1924) was widely accepted (Bouchet \& Rocroi 2005, Williams et al. 2010). Members of Calliostomatidae occur nearly worldwide, exhibiting physical and habitat diversity. Comments regarding diet, reproduction, habitat and shell appearance are specific to those species of the northeast Pacific.

The northeast Pacific spans multiple marine provinces (MP) including, the Arctic, Oregonian-Alaskan, and Californian, which collectively encompasses coastal Alaska (USA), British Columbia (Canada), Washington, Oregon, California (USA), and Baja California (Mexico).

Four genera of Calliostomatidae are currently recognized from the northeast Pacific:

Akoya Habe, 1961. White shelled species occurring in moderately deep water from Alaska to southern California, with conical shape, low cords and threads beaded/not beaded.

Calliostoma Swainson, 1840. Species with variegated patterns with threads/cords often beaded, shells cream brown, gray, soft yellow, often with multi colored peripheral band, flammules or blotches.

Otukaia Ikebe, 1942. White shelled species occurring in moderately deep water in the Bering Sea, with large strongly keeled cords may lack beading.

Xeniostoma McLean, 2012. Small species with a white shell lacking threads, cords, or carination, umbilicus open, from moderately deep water off Alaska.

The goal of this contribution is to facilitate the identification of Calliostomatidae from the northeast Pacific and to compile past and current literature relevant to their identity. Distinctive shell characteristics are provided to aid in identification and are not re-descriptions of named taxa. Approximately one-third of the calliostomids in the northeast Pacific occur most frequently below 75-100 m, the remainder may be found intertidally or while SCUBA diving. As deep-water surveys continue, it is expected that the known range of numerous species will be expanded. Many species prevalent in the Oregonian MP and further north are less abundant in the Californian MP and often at greater depths where the water is cooler. The temperate Californian MP also contains some tropical species typical of the Panamic MP. Otukaia and Xeniostoma are both deep-water genera. At present, Xeniostoma is a monotypic genus. 


\title{
Materials and Methods
}

The collection of the San Diego Natural History Museum (SDMNH), Natural History Museum of Los Angeles County (LACM), Santa Barbara Museum of Natural History (SBMNH), the Benthic Collection at Scripps Institute of Oceanography (SIO), City of San Diego Ocean Monitoring Program (CSDOMP), Carnegie Museum of Natural History (CMNH), National Museum of Natural History, Smithsonian (USNM) were visited and various private collections examined. The computerized collection database of the California Academy of Science (CAS, CASIZ), Academy of Natural Sciences, Philadelphia (ANSP), and the Natural History Museum of London (NHMUK), were also reviewed. In addition, the author has SCUBA-dived and studied Calliostoma in the Oregonian, Californian, and Panamic marine provinces.

\author{
Abbreviations \\ H/W Height and width measurements \\ MP Marine Province
}

\section{Systematics}

Calliostoma Swainson, 1840

Calliostoma Swainson, 1840: 218, 351. Type species (SD Herrmannsen 1846: 154): Trochus conulus Linnaeus, 1758. Europe.

Description. Shell moderately high-spired, whorls flat-sided or rounded; final lip not thickened. Peristome incomplete, columella lacking folds or denticles, somewhat thickened, truncate toward base, often with external oblique columella scar or sulcus. Early sculpture beaded spiral threads/cords, beading persisting or lost in later growth stages. Protoconch with reticulate sculpture, rounded pits. Color patterns often variegated; interior iridescent, exterior surface may show metallic luster. Calliostoma of northeast Pacific without open umbilicus or sulcus, but rather slight scar-like shallow depression.

Remarks. Calliostoma of the northeast Pacific are opportunistic feeders on a range of soft bodied sessile invertebrates and are occasional scavengers, allowing them to occur in varied habitats and depths compared to the Eastern Pacific Trochidae that consume algae. Prey species include bryozoans, hydrozoans, sponges, diatoms, and various cnidarians including the anemone Corynactis californica (Carlgren, 1936), hydrocoral Stylaster californica (Verrill, 1866), and gorgonians (Perron 1975, Keen 1975, Harbo 2007, Morris et al. 1990, Stone et al. 2014, Tuskes \& Tuskes 2019). Three species [C. annulatum (Lightfoot, 1786), C. canaliculatum (Lightfoot, 1786), C. ligatum (Gould, 1849)], may be found on large brown kelp and consume the fauna that lives on the fronds and periodically the fronds or reproductive growth. See Tuskes \& Tuskes (2019) for additional details regarding the diet of specific species and other biological information. The reproductive biology of C. ligatum, as a broadcast spawner is similar to Tegula in the family Trochidae; for details on development see Holyoak (1988).

Species Excluded. A single specimen of Calliostoma antonii (Koch, 1843), USNM 206116, is labeled San Diego, California "Cooper". That record is doubtful as no other California material has been found, and McLean (1971) gave the distribution of C. antonii as El Salvador to Peru.

\section{Calliostoma annulatum (Lightfoot, 1786)}

\section{(Figure 1)}

Trochus annulatus "Martyn," Lightfoot, 1786: 101. Type whereabouts not known. Type locality not designated.

Trochus annulatus Martyn, 1784: pl. 33 [unavailable]. ICZN (1957: opinion 456).

Trochus virgineus Chemnitz, 1788: 10: 289, pl. 165, figs 1581, 1582 [unavailable]. ICZN (1954: direction 1).

Trochus rubiginosus Valenciennes, 1846: pl. 4, figs 1, 1a, 1b. Type whereabouts unknown. Type locality not designated.

Description. Shell relatively thin, height $20-35 \mathrm{~mm}$. Ground color golden yellow, apex purple; whorls 
slightly convex; threads golden-yellow, lightly beaded; cords golden-yellow, bead color alternating light yellow, red/brown; peripheral band bright purple; base rounded, basal threads 7-10 beaded, purple, band extends from lip, wraps around columella into shell; columella scar iridescent silver.

Distribution. The northern record for this species is east of Afognak Island, Alaska, $58^{\circ} 18.13^{\prime} \mathrm{N}$, $151^{\circ} 09.93^{\prime} \mathrm{W}$ (Clark 2018). The southern record is currently San Geronimo Island, Baja California $\left(30^{\circ} \mathrm{N}\right)$. Found on rocky bottoms, Macrocystis kelp and other large kelp species. Locally common north of Point Conception, Santa Barbara County, California, uncommon south of Point Conception. Lower intertidal to 45 $\mathrm{m}$.

Remarks. Old museum material fades such that the purple band and beading becomes drab yellowbrown. Small faded shells appear similar to faded $C$. variegatum. The whorls of $C$. annulatum are convex and beads are uniform in size; whorls of $C$. variegatum are flat, threads have smaller beads than those on cords.

\section{Calliostoma canaliculatum (Lightfoot, 1786)}

(Figure 2)

Trochus canaliculatus "Martyn” Lightfoot, 1786: 101. Type whereabouts unknown. Type locality not designated. Trochus canaliculatus Martyn, 1784: pl. 42 [unavailable]. ICZN (1957: opinion 456).

Trochus doliarius Chemnitz, 1788: 10: 288, pl. 165, figs 1579, 1580 [unavailable]. ICZN (1954: direction 1).

Description. Shell relatively thin, height $20-45 \mathrm{~mm}$. Ground color tan; whorls flat-sided, spiral cords prominent, color off-white, smooth, beading vestigial if present; interspace tan-brown; flammules when present brown; base angulate, basal cords 5-6 smooth, threads 4-5 smooth, columella scar iridescent purplegray.

Distribution. Sitka, southeastern Alaska $\left(57^{\circ} \mathrm{N}\right)$, to San Benito Island $\left(28^{\circ} \mathrm{N}\right)$, Baja California. Locally common north of Point Conception, Santa Barbara County, California, lower intertidal to $90 \mathrm{~m}$, found on kelp fronds and hard structure. Uncommon south of Point Conception.

Remarks. The largest Calliostoma species in the northeast Pacific. Both C. canaliculatum and C. ligatum have strong smooth cords. The whorls of $C$. ligatum are bulbous, and the base is rounded, while whorls of $C$. canaliculatum are nearly flat, and the base angulate.

\section{Calliostoma eximium (Reeve, 1842)}

(Figure 3)

Trochus eximius Reeve, 1842a: 185; Reeve (1842b: pl. 118, fig. 12). Holotype: Museum Cuming: not at NHMUK. Payanum, Panama Bay, Panama, muddy sand, $10 \mathrm{fm}$ (18.3 m).

Trochus versicolor Menke, 1850: 172-173. Holotype whereabouts unknown. Mazatlan.

Description. Shell sturdy, height 20-27 mm. Ground color gray-yellow to light yellow; whorls concave above, shoulder convex; periphery flat, shoulder sloping tabulate; threads low, beaded, dark brown dashes form broken lines; body whorl flammules, when present, gray-brown, vary in intensity; base rounded, basal threads shallow/alternating light brown-orange spots, columella/scar metallic silver.

Distribution. Cedros Island $\left(28^{\circ} \mathrm{N}\right)$, Baja California, Gulf of California, south to Peru $\left(10^{\circ} \mathrm{S}\right)$. Specimens have been collected intertidally on muddy sand and to depths of 50-60 m. Locally common.

Remarks. Both Dall (1921) and Oldroyd (1927) listed Santa Catalina Island off southern California as the northern extent of the range. Reeve (1842a, 1842b) was not the source, as he only mentions the type locality in Panama. One dubious specimen is in the SDNHM from La Jolla (San Diego), but the data card with the same number as the shell lacked both date and collector and was for a series of C. tricolor.

\section{Calliostoma gemmulatum Carpenter, 1864}

(Figures 4, 26)

Calliostoma formosum Carpenter, 1864b: 156. Holotype USNM 16261. San Pedro, California. (unavailable, non McAndrew \& Forbes, 1847)

Calliostoma gemmulatum Carpenter, 1864a: 215 [new name for C. formosum Carpenter].

Margarites keepi Smith \& Gordon, 1948: 228-229, pl. 4, figs 5-7. Holotype CAS Paleo 8557. Monterey Bay, 25 fm. 


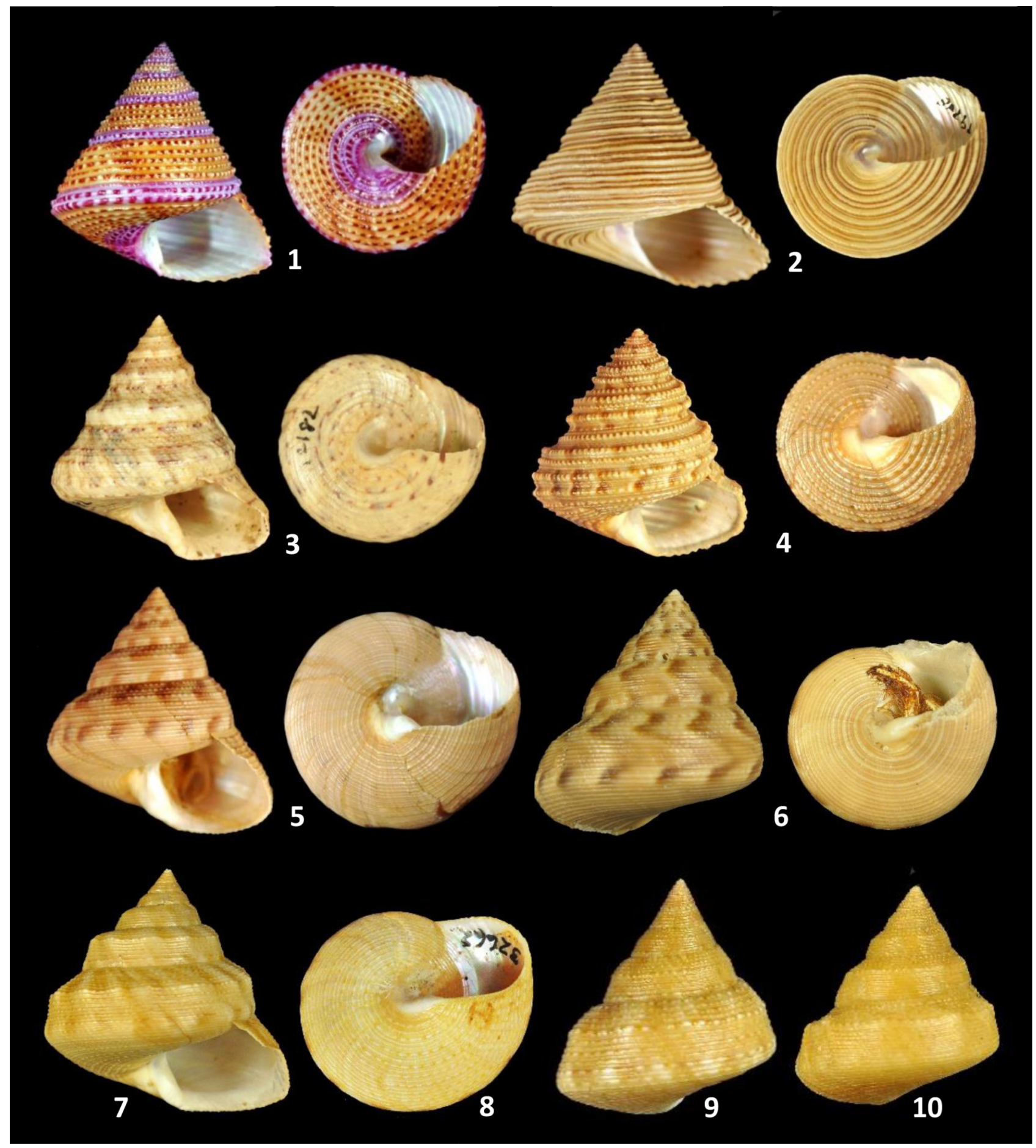

FIGURES 1-10. 1. Calliostoma annulatum $27.1 \mathrm{~mm}$, Monterey Bay, Monterey County, California, (LACM 181622). 2. Calliostoma canaliculatum $37.2 \mathrm{~mm}$, on floating kelp, Monterey Bay, Monterey County, California (LACM 19748). 3. Calliostoma eximium $26.7 \mathrm{~mm}$, Scammons Lagoon, Baja California (SDMNH 2903). 4. Calliostoma gemmulatum $17.6 \mathrm{~mm}$, San Pedro, Los Angeles County, California (LACM 23418). 5. Calliostoma gloriosum 28.4 mm, San Pedro, Los Angeles County, California, (LACM 57752). 6. Calliostoma gloriosum $21.2 \mathrm{~mm}$, Sacramento Reef, south of Geronimo Island, Baja California (LACM 3640). 7. Calliostoma guerreroensis holotype (LACM 3640), 32.4 mm, off Magdalena Bay, Baja California. 8-9. Calliostoma guerreroensis paratypes, off Laguna Guerrero Negro, Baja California (SBMNH 132662). 8. 23.6 mm. 9. 15.9 mm. 10. Calliostoma guerreroensis paratype. $14.9 \mathrm{~mm}$, subadult, off Natividad Island, Baja California, (LACM 71-165.10). 
Description. Shell thin, height 13-21 mm. Ground color brown, vertical streaks/blotches brown/olive; whorls convex, suture deeply impressed; body whorl, three cords strongly beaded, interspaced low beaded thread 23 , shoulder multiple fine threads, minutely beaded; base angular, basal cords 10-12, alternating light brownorange spots; beading absent/weak, columella scar metallic white.

Distribution. San Simeon (CM 142913), San Luis Obispo County, California (35N), to San Juanico Bay $\left(26^{\circ} \mathrm{N}\right)$, Baja California Sur. Intertidal to $100 \mathrm{~m}$. Rocky habitats along the open coast and rocky entries to bays. Often not collected for intervals of several years. Uncommon.

Remarks. Margarites keepi was based upon a juvenile shell $2 \mathrm{~mm}$ in height taken in Monterey Bay, California $\left(38^{\circ} \mathrm{N}\right)$; mature specimens have not been collected that far north.

\section{Calliostoma gloriosum Dall, 1871}

(Figures 5-6, 27)

Calliostoma gloriosum Dall, 1871: 127. Holotype USNM 206134. Monterey Bay, California.

Description. Shell sturdy, height $20-35 \mathrm{~mm}$. Ground color dull orange, tan, to gray-brown; whorls lightly convex, body whorl, threads finely beaded below suture, peripheral threads not beaded; flammules light/dark brown or orange-brown; base angular; basal threads fine, not beaded; columella scar silver-white.

Distribution. Calliostoma gloriosum occurs from San Francisco, California $\left(38^{\circ} \mathrm{N}\right)$, to at least San Benito Island, $\left(27^{\circ} \mathrm{N}\right)$, Baja California. Common north of Point Conception, Santa Barbara County, California, occurring from the lower rocky intertidal to $100 \mathrm{~m}$. Uncommon south of Point Conception, California, 5-100 m.

Remarks. A small percentage of shells have notably bulbous whorls. Calliostoma gloriosum has been confused with Calliostoma guerreroensis, see below.

\section{Calliostoma guerreroensis Tuskes \& Tuskes, 2019}

(Figures 7-10)

Calliostoma guerreroensis Tuskes \& Tuskes, 2019: 11, figs 7-10. Holotype LACM 3640. $24-27 \mathrm{~m}, 5.45 \mathrm{mi} .230^{\circ} \mathrm{T}$, from Punta Entrada, Bahia Magdalena, Baja California Sur, Mexico (243․'N, $\left.112^{\circ} 01.00^{\prime} \mathrm{W}\right)$. Leg. R/V Velero IV 1971-50 (LACM 50-41).

Description. Shell sturdy, height 25-35 mm; ground color light yellow-brown (straw). Bodywhorl, shoulder with 5-6 beaded cords, lightly beaded thread between cord; distinctive axial ridges, diagonal flammules alternating brown/cream, raised/not raised; below last prominent cord of shoulder angle abruptly changing from approximately 35 degrees to approximately 80-90 degrees; lateral surface with 3-4 beaded cords alternating cream/straw, threads between cords smooth/low profile straw beads; peripheral band, three cords, alternating brown square with larger cream rectangular markings, cords beaded, alternating cream/straw; base with 16-18 shallow straw cords with/without low beading, interspace threads, cords proximal to columella broader, numerous cords with alternating cream/straw spots present; columella white, shallow columellar scar white to lustrous.

Distribution. From Cedros Island, Baja California to Magdalena Bay, Baja California Sur. Depth 27$55 \mathrm{~m}$. Uncommon.

Remarks. The unique axial ridges on body whorls 6-7 are not present on smaller shells. The intensity of the peripheral bands is variable and may not be notable in juveniles. The ground color may be slightly darker among juveniles. The number of basal cords with alternating cream/light brown spots is variable but always present. Juvenile shells typically lack the distinct pattern of mature shells. Shells of subadults have the shape and markings of adults, but lack bulk. This species is easily distinguished by its stepped profile and alternating cream/light brown spots on basal cords; C. gloriosum (Figs 5-6) lacks these characteristics and has a rounded profile.

\section{Calliostoma keenae McLean, 1970}

(Figure 11)

Calliostoma keenae McLean, 1970: 424, pl. 62, figs 13, 14. Holotype LACM 1272. Off Laguna Beach, Orange County, California, 106-109 m. 
Description. Shell thin, height $10-15 \mathrm{~mm}$. Ground color drab yellow; whorls convex, shoulder sloping tabulate, threads below suture beaded, threads immediately above, below periphery band, typically smooth; flammules light brown; peripheral band alternating dark brown/white. Base rounded, basal threads nearly smooth; columella scar light brown. Shell coloration fades with age.

Distribution. Laguna Beach, Orange County, California $\left(34^{\circ} \mathrm{N}\right)$, to Cape San Lucas, Baja California Sur, south to Clarion Island $\left(18^{\circ} \mathrm{N}\right)$ in the Revillagigedo group. Skoglund \& Koch (1993) reported this species in the Gulf of California, as far north as Los Angeles Bay, depth 45-110 m. Rare.

\section{Calliostoma ligatum (Gould, 1849)}

(Figures 12, 28)

Trochus ligatus Gould, 1849: 91; 1856: pl. 12, fig. 207. Holotype USNM 5608. Puget Sound, Washington.

Trochus costatus Martyn, 1784: pl. 34 [unavailable ICZN (1957: opinion 456), not T. costatus Gmelin, 1791]. Holotype whereabouts unknown. No type locality designated.

Trochus filosus Wood, 1828: 17, 56 [not Helbling, 1779]. Holotype whereabouts unknown. Type locality not designated.

Trochus modestus Middendorff, 1849: 413, pl. 10, figs 16-18 [not Reeve, 1842, nor Koch, 1845]. Holotype whereabouts unknown. Sitka Island, Alaska.

Trochus middendorfii Philippi, 1851: 337 [new name for T. modestus Middendorff].

Trochus castaneus "Nuttall," Forbes, 1852: 271. Holotype whereabouts unknown. Upper California.

Calliostoma costatum caeruleum Dall, 1919: 359. Syntype USNM 59808. Monterey, Monterey County, California.

Calliostoma costatum pictum Dall, 1919: 359. Syntype USNM 12612a. Neah Bay, Clallam County, Washington.

Description. Shell sturdy, height $17-30 \mathrm{~mm}$. Ground color brown-light brown; whorls bulbus; early spiral cords smooth/rarely lightly-beaded; lower whorls, cords unbeaded, light brown-off white; interspaces broader than cords, brown-light brown; base rounded, basal cords smooth (8-12) light brown; columella scar metallic white.

Distribution. Afognak Island, Alaska $\left(58^{\circ} \mathrm{N}\right)$, to San Benito Island, Baja California $\left(28^{\circ} \mathrm{N}\right)$. Locally common in rocky intertidal to $30 \mathrm{~m}$. South of Point Conception, Santa Barbara County, California, found at 20-95 m on hard substrate. Scarce.

Remarks. There are two color forms, caeruleum has a sub-sutural purple band and form pictum has brown flammules. The purple band of $C$. ligatum often fades completely within 1-2 years after collection. Purple-ringed $C$. ligatum are easily separated from $C$. annulatum, by examining the cords, $C$. ligatum has smooth cords while those of $C$. annulatum are strongly beaded. Also see C. canaliculatum.

\section{Calliostoma nepheloide Dall, 1913}

(Figures 13, 29)

Calliostoma nepheloide Dall, 1913: 592. Two syntype USNM 96637. Panama Bay, Panama, 86 m.

Description. Shell thin, height $15-28 \mathrm{~mm}$. Ground color olive-brown to yellow-brown; whorls flat; two prominent beaded peripheral cords; threads numerous, shallow, beaded lightly, interspace metallic copper/ light brown; flammules dark purple-brown/absent; peripheral band alternating white/brown; base angulate, basal threads 17-22 shallow, beaded; columella scar metallic white/silver.

Distribution. Point Abreojos $\left(27^{\circ} \mathrm{N}\right)$, Baja California Sur, to Panama $\left(18^{\circ} \mathrm{N}\right)$. McLean $(1971)$ gave a depth of 73-120 m, which is consistent with data on museum material. Houston (1980) indicated the species was common in Baja California Sur in both estuaries and mangroves stands with sandy-mud bottoms and found intertidally; although contrary to museum records it may represent a previously unidentified aspect of its biology.

Remarks. This species has been taken in deep water in the northern transition zone between the Californian and Panamic MP and is included because there is a reasonable possibility that it will be found within the geographic scope of this publication series. 


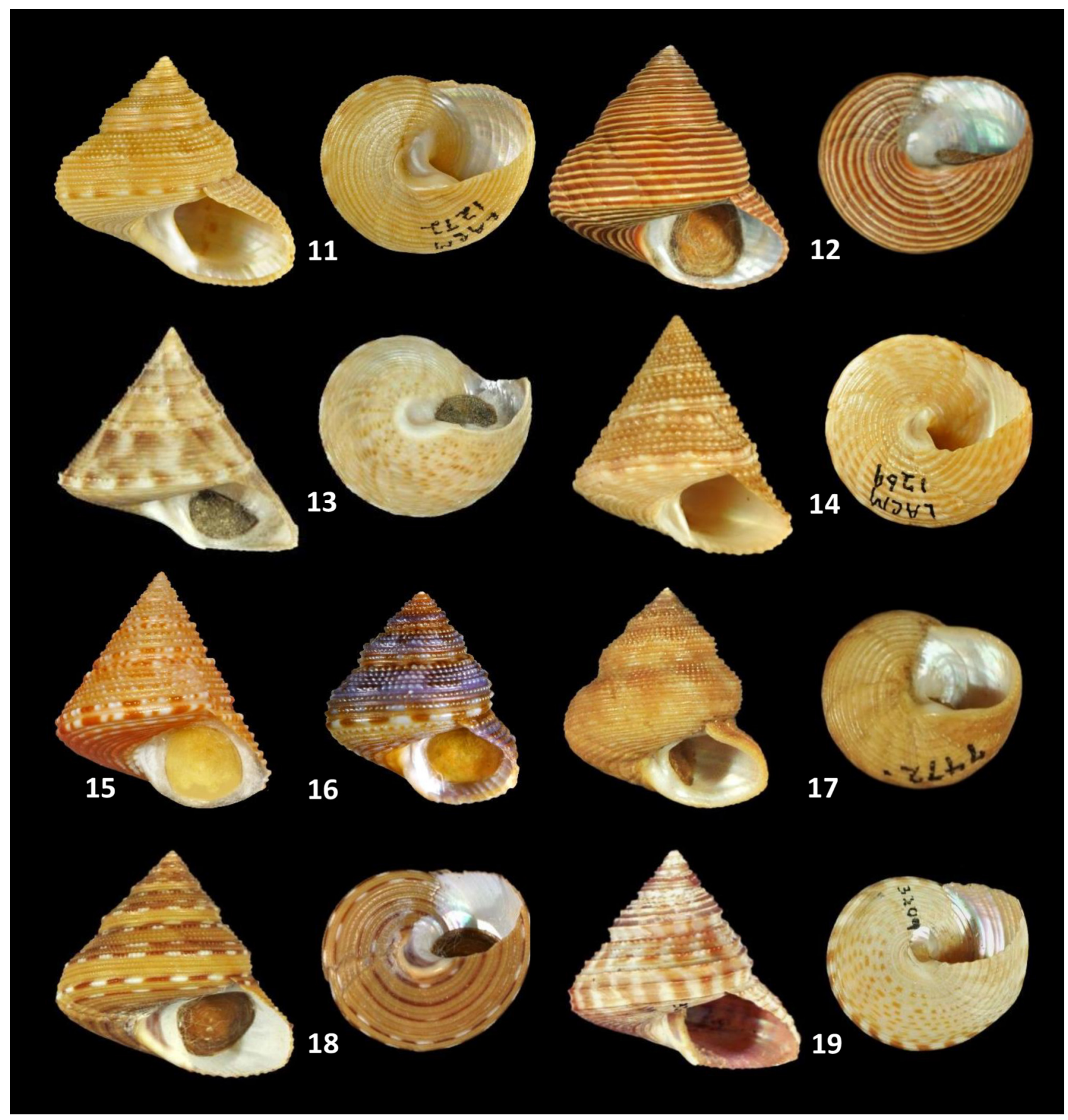

FIGURES 11-19. 11. Calliostoma keenae holotype (LACM 1272), 15.6 mm, Laguna Beach, Los Angeles County, California. 12. Calliostoma ligatum $26.2 \mathrm{~mm}$, Morro Bay, San Luis Obispo County, California (LACM 181023). 13. Calliostoma nepheloide $15.6 \mathrm{~mm}$, Chiriqui, Golfo de Chirqui, Panama (SBNHM 157918). 14. Calliostoma sanjaimense holotype (LACM 1263), $20.0 \mathrm{~mm}$. San Jaime Bank off Cape San Lucas, Baja California 15. Calliostoma sanjaimense $16 \mathrm{~mm}$, Nine Mile Bank off San Diego, San Diego County, California (LaGrange collection). 16. Calliostoma supragranosum 8 mm, Mission Bay Jetty, San Diego, San Diego County, California (Tuskes collection). 17. Calliostoma supragrenosum 12.9 mm, San Pedro Bay, Los Angeles County, California (SBMNH 7772). 18. Calliostoma tricolor $21.5 \mathrm{~mm}$, White Point, Los Angeles County, California (SBMNH 16907). 19. Calliostoma turbinum 17.3 mm, La Jolla, San Diego County, California, 183 m (SBMNH 103209). 
Calliostoma sanjaimense McLean, 1970

(Figures 14-15)

Calliostoma sanjaimense McLean, 1970: 423, pl. 62, fig. 11. Holotype LACM 1269. San Jaime Bank, Baja California Sur, 137 $\mathrm{m}$.

Description. Shell small; height $10-16 \mathrm{~mm}$. Ground color dark yellow; whorls flat; texture rough; two peripheral cords strongly developed, beaded, alternately marked white/orange-brown; threads dark yellow, beaded dark yellow/brown; base angulate, 9-11 cords nearly smooth; columella metallic white.

Distribution. A deep-water species from San Diego $\left(33^{\circ} \mathrm{N}\right), 140-160 \mathrm{~m}$ (LaGrange 1992), south to Cape San Lucas $\left(23^{\circ} \mathrm{N}\right)$, Baja California Sur. Rare.

Remarks. The holotype has faded.

\section{Calliostoma supragranosum Carpenter, 1864c}

(Figures 16-17, 30)

Calliostoma supragranosum Carpenter, 1864c: 653. Holotype USNM 14925 (Palmer 1958: pl. 17, figs 14, 15). San Diego, California.

Calliostoma splendens Carpenter, 1864b: 156. Syntype USNM 16278 (Palmer 1958: pl. 17, figs 11, 12). Santa Catalina Island, California.

Description. Shell thin, height 8-14 $\mathrm{mm}$. Ground color reddish-brown whorls convex; body whorl threads beaded above periphery, beading light/absent below periphery, flammules present/absent darker than ground color; peripheral band alternating brown, white patches, weak/strong; base rounded, basal cords 7-8 smooth; columella scar metallic white-green-blue.

Distribution. Cordell Bank, off Point Reyes, Marin County, California $\left(38^{\circ} \mathrm{N}\right)$, to Asuncion Island $\left(27^{\circ} \mathrm{N}\right)$, Baja California Sur. Occurs in rocky subtidal habitats to $30 \mathrm{~m}$. Uncommon.

Remarks. The purple-blue phenotype is rare. Both specimens illustrated have undulations on the inner lip, but are only obvious on the blue specimen because of the contrasting colors. Calliostoma splendens has been treated as a synonym since McLean (1969). For details and past usage see Tuskes \& Tuskes (2019). Most specimens taken in recent years average $10 \mathrm{~mm}$ or less in height, old collections contain specimens to $14 \mathrm{~mm}$.

\section{Calliostoma tricolor Gabb, 1865}

(Figure 18)

Calliostoma tricolor Gabb, 1865: 186. Syntype ANSP 38184. San Pedro, California.

Description. Shell solid, height $15-22 \mathrm{~mm}$. Ground color dull yellow-orange; whorls slightly bulbus, slightly concave, rounded periphery, suture impressed; threads beaded, color variable: yellow, alternately white/dark brown, alternating yellow-white; base angulate, basal threads 8-11 lightly beaded/unbeaded; columella scar slightly depressed, gray-blue distal, metallic silver proximal.

Distribution. Half Moon Bay, San Mateo County, California $\left(38^{\circ} \mathrm{N}\right)$, to Tortugas Bay, $\left(28^{\circ} \mathrm{N}\right)$, Baja California Sur, Mexico. North of Point Conception, Santa Barbara County, California this species may be found intertidally, but occurs below the thermocline in southern California, 25-70 m. Locally common north of Point Conception, uncommon to south.

Remarks. Gabb (1865) did not designate a holotype in the original description. Coan \& Bogen (1988) located a syntype in the ANSP collection. After reviewing various photos of that specimen and discussions with the collection manager, I believe it is likely the post Pliocene specimen, which Gabb mentioned in the original description along with other recently collect material from California. The syntype is eroded and lacks many of the diagnostic features, but the written description of $C$. tricolor is appropriate for current material. 

Conception, Santa Barbara County, California, $183 \mathrm{~m}$.

Description. Shell thin, height 15-20 mm. Ground color metallic silver-gray: whorls lightly convex; spiral threads beaded, interspace iridescent metallic copper; flammules narrow, elongated, numerous, iridescent copper, lower peripheral flammules extend to basal margin; base rounded, basal threads/cords heavily marked, 10-12 copper spots; columella scar iridescent white proximally, faint gray-blue distally. When rotated under light the colors change, iridescent copper may become brown.

Distribution. Point Conception, Santa Barbara County, California $\left(34^{\circ} \mathrm{N}\right)$, to San Benito Island, Baja California $\left(28^{\circ} \mathrm{N}\right)$. Depth usually $90-180 \mathrm{~m}$, rarely as shallow as $26 \mathrm{~m}$. Scarce.

\section{Calliostoma variegatum Carpenter, 1865}

(Figure 20,32)

Calliostoma variegatum Carpenter, 1865: 61. Holotype USNM 4201 (Palmer 1958: pl. 17, figs 9, 10). Puget Sound, Washington.

Description. Shell sturdy, height $18-29 \mathrm{~mm}$. Ground color light yellow to light yellow-tan, apex rose, whorls flat, suture slightly impressed, threads heavy beaded, alternating yellow/orange/brown; base angular, basal cords $7-8$, beaded, alternating cream and light orange spots, simple threads between some cords, ground color; columella scar pearl; aperture crenulate.

Distribution. East of Afognak Island, Alaska, $58^{\circ} 18.13^{\prime} \mathrm{N}, 151^{\circ} 09.93^{\prime} \mathrm{W}$ (Clark 2018), south to Cedros Island, Baja California $\left(28^{\circ} \mathrm{N}\right)$. Taken by SCUBA divers in the northern portion of range on soft bottoms, southern portion of the range found at $70-230 \mathrm{~m}$. Scarce.

Remarks. The shell may fade to a uniform dull yellow and may be mistaken for small faded $C$. annulatum. Whorls of $C$. annulatum are convex and beads are uniform in size; whorls of $C$. variegatum are flat and threads have smaller beads than those on cords. Semi-alba shells are known, white with rose apex.

\section{Akoya Habe, 1961}

Akoya Habe, 1961: 10. Type species (OD): Calliostoma akoya Kuroda in Ikebe, 1942.

Description. Shell thin, height 30-37 mm; ground color white or iridescent silvery-white; periostracum semitransparent gray-brown; whorls convex; spiral beaded cords usually present on early teleoconch; whorls with spiral cords/threads beaded/unbeaded, becoming frequent/obsolete on later whorls; columella and scar white.

Remarks. The status of Akoya has fluctuated. It is treated here as a genus, acknowledging a wide-ranging group of deep-water white shells with the general conformation of both Calliostoma and Otukaia characteristics. At the moment, the lineage of deep-water white shells is elusive. As methods of genetic evaluation become both more sophisticated and comprehensive our understanding of clades may definitively address evolutionary trends and better define genera. Ikebe (1942) was the author of a journal article in which Kuroda described Calliostoma akoya. As such, Ikebe is the author of the publication while Kuroda in Ikebe is the taxon authority.

\section{Akoya platinum (Dall, 1890)}

(Figures 22-24, 33)

Calliostoma platinum Dall, 1890: 343, pl. 7, fig. 7. Holotype USNM 96558. R/V Albatross Station 2839, near Santa Barbara Island, California, $756 \mathrm{~m}$.

Calliostoma titanium McLean, 1984: 236, fig. 4. Holotype LACM 1995. R/V Velero 1027-39 8.3 km SE of Santa Catalina Island, California, 256-274 m.

Calliostoma bernardi McLean, 1984: 236, figs 5, 6. Holotype: LACM 1996. R/V Velero 1152-40SE end of Santa Catalina Island, California, 241-271 m. 


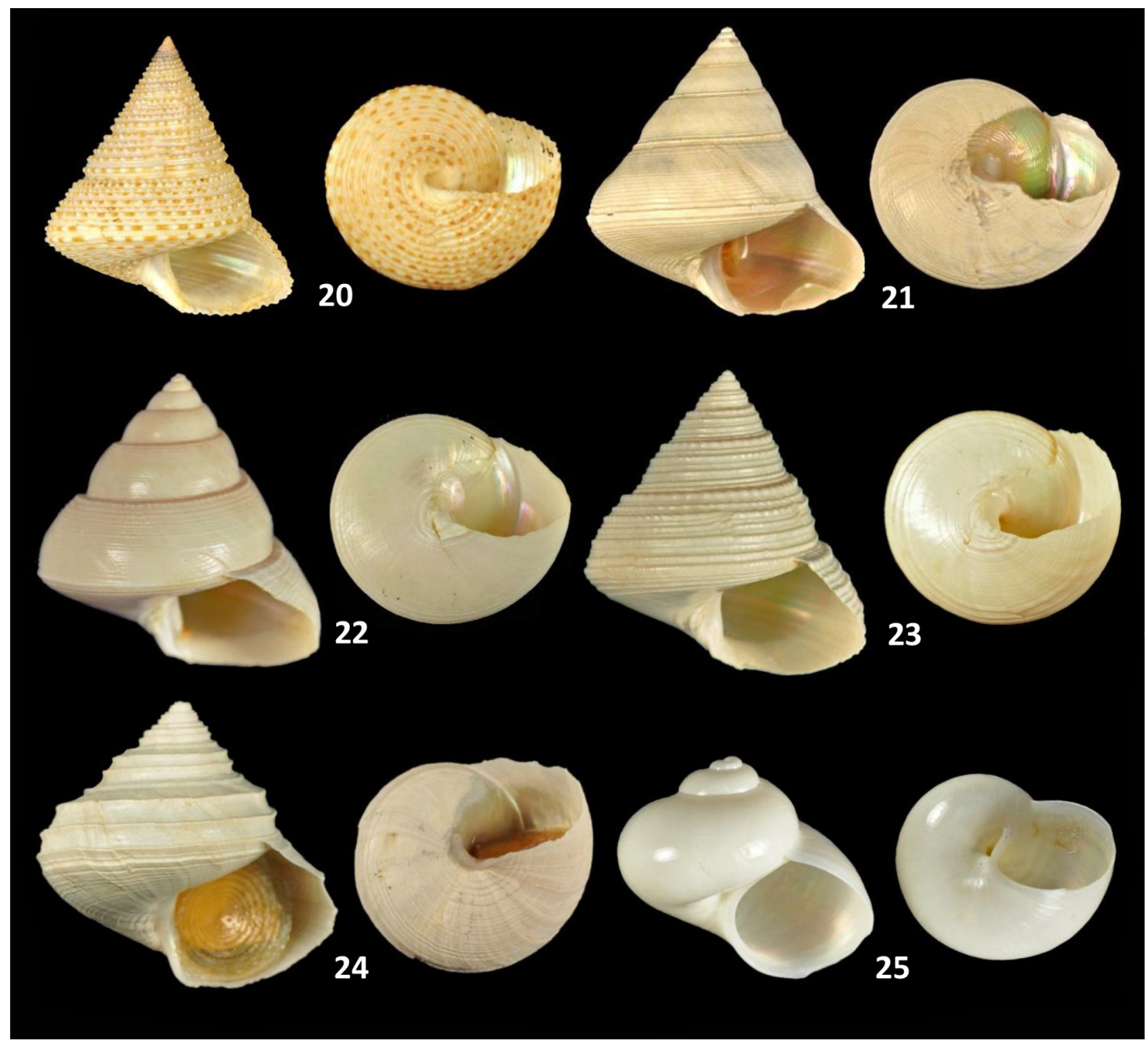

FIGURES 19-25. 20. Calliostoma variegatum $25.9 \mathrm{~mm}$, Forrester Island, Prince of Whales County, Alaska (LACM 163523). 21. Akoya platinum $29 \mathrm{~mm}$, lip damaged. Tanaga Island, Bering Sea, Aleutians West County, Alaska, 1,247 m (LACM 179493). 22. Calliostoma titanium holotype (LACM 1995) $32.2 \mathrm{~mm}, 8.3 \mathrm{~km}$ SE of Santa Catalina Island, California, 256-274 m. 23. Calliostoma bernardi holotype (LACM 1996) $26.6 \mathrm{~mm}$, SE end of Santa Catalina Island, California, 241-271 m. 24. Otukaia beringensis holotype (LACM 3514) $26.5 \mathrm{~mm}$, west central Aleutian Islands, Alaska, 1,247 m. 25. Xeniostoma inexpectans holotype (LACM 2471) 8.7 mm. 272 miles west of Kiska Island, Rat Islands, Aleutian Islands, Alaska, 219-384 $\mathrm{m}$.

Description. Shell thin, height 25-37 mm. Ground color white (periostracum removed); whorls convex; teleoconch 2-3 unbeaded threads; whorls, subsutural threads $0-5$ weak/absent, threads smooth rarely lightly beaded; peripheral cords 1-3 smooth; threads immediate above/below, absent/microscopic; base angular, basal threads 35+ absent/minute; columella scar pearl-sliver; notable carina present/absent adjacent to columella.

Distribution. From Icy Bay, Gulf of Alaska $\left(59^{\circ} 17.61^{\prime} \mathrm{N}, 141^{\circ} 56.04^{\prime} \mathrm{W}\right.$ : Clark 2018), south to San Diego $\left(33^{\circ} \mathrm{N}\right)$, California. $180-700 \mathrm{~m}$. Akoya platinum undoubtedly occurs south of San Diego, California, at appropriate depth along the northern Baja California peninsula. Scarce.

Remarks. The shells illustrated represent the ideal phenotypes for A. platinum, form titanium and form bernardi. Variation in color (gray/gray-brown vs white results from the presence or absence of the 
periostracum. Tuskes \& Tuskes (2019) illustrated the intermediate forms and provide additional quantitative results. Forms titanium and bernardi have progressively increasing number of cords/threads and beading.

Otukaia Ikebe, 1942

Otukaia Ikebe, 1942: 277. Type species (OD): Calliostoma kiheiziebisu Otsuka, 1939. Japan.

Description. Shell moderate to large; ground color silky-white to light tan, shell thin; two or more spiral cords present on early teleoconch whorls, becoming obsolete on later whorls of some species, retained in others; spiral cords beaded in some species, smooth, broad/angular in others.

Remarks. This group had been considered to be broadly distributed in moderately deep water, but many species have since been transferred into other genera of Calliostomatidae. The type species from Japan was figured by Sasaki (2000). In addition to Japan, O. kiheiziebisu has been found at 1,200 $\mathrm{m}$ off Hawaii (Severns 2011).

\section{Otukaia beringensis Tuskes \& Clark, 2018}

(Figure 24)

Otukaia beringensis Tuskes \& Clark, 2018: 236, figs 1-2. LACM 3514. Central Aleutian Islands, $52^{\circ} 52^{\prime} \mathrm{N}, 178^{\circ} 17^{\prime} \mathrm{W}$, Alaska, $1247 \mathrm{~m}$.

Description. Shell thin, height $25-35 \mathrm{~mm}$. Ground color white; body whorl, two projecting keeled cords, above peripheral; one secondary keeled cord below suture, peripheral cord slightly keeled, sub-peripheral cord low, broadly rounded; base, 25+ finely spaced shallow threads, proximal to columella broadly flattened, centrally narrow, distally slightly raised, some incomplete; columella/basal scar dull white; umbilicus closed aperture oval; operculum circular, light brown,

Distribution. All specimens are from the Eastern Bering Sea. It is not known if the Aleutian Basin or the Aleutian Trench, which are deeper than the current known locations, affect their distribution.

Remarks. This is the first species of Otukaia to be collected in the northeast Pacific. The current known depth range is 818 to $1,247 \mathrm{~m}$, with additional sampling, both depth and distribution will be better defined. The holotype was photographed by Robert Stone during his research via an ROV. The animal was feeding on the gorgonian Calcigorgia beringi (Nutting, 1912) (R. Stone pers. comm.) and a paratype was associated with bamboo coral Isidella sp., which is also a gorgonian.

\section{Xeniostoma McLean, 2012}

Xeniostoma McLean, 2012: 89. Type species (OD) Xeniostoma inexpectans. Aleutian Islands, Alaska.

Description. Shell small to moderate, rounded, smooth lacking spiral threads or cords, height 8-12 mm; protoconch smooth and large, nearly $1 \mathrm{~mm}$.

Remarks. Currently it is a monotypic genus. The lack of enlarged inner marginal teeth distinguishes this subfamily (Xeniostomatinae) from typical members of the subfamily Calliostomatinae. The lack of any spiral sculpture, the lack of jaws, and the lack of the pseudoproboscis are further differences from Calliostomatinae. Other recent or fossil material awaiting discovery will further define the characters of this recently described genus and subfamily.

\section{Xeniostoma inexpectans McLean, 2012}

(Figure 25)

Xeniostoma inexpectans McLean, 2012: 89, figs 1-6. Holotype LACM 2471. West of Kiska Island, Rat Islands, Aleutian Islands, Alaska $\left(51^{\circ} 54.31^{\prime} \mathrm{N}, 176^{\circ} 35.89^{\prime} \mathrm{E}\right) .272 \mathrm{~m}$.

Description. Shell thin, height 8-12 mm. Ground color white interior strongly iridescent; shell slightly broader than high; texture smooth lacking spiral or axial sculpture; base rounded; umbilicus open; columella and scar white.

Distribution. The species is known from the eastern portion of the Rat Islands. This group of around 15 
islands is in the western portions of the Aleutian Island chain of Alaska. The species has been taken at depths of 219-384 m. A large sample of live specimens were collected off south central Oregon coast $\left(43^{\circ} 27^{\circ} 0^{\prime \prime} \mathrm{N}\right.$, $124^{\circ} 52^{\prime} 34^{\prime \prime} \mathrm{W}$ ) in 1974 by Oregon State University (SBMNH 470508) at a depth of $630 \mathrm{~m}$. The disjunct distribution is likely due to lack of adequate sampling. Scarce.

Remarks. This species is found associated with a vase shaped hexactinellid sponge in the family Rossellidae. The lack of jaws and the unique radula suggest it consumes detritus that collects in the bowl of the sponge. There is no indication it feeds on the tissue of the sponge (McLean 2012).

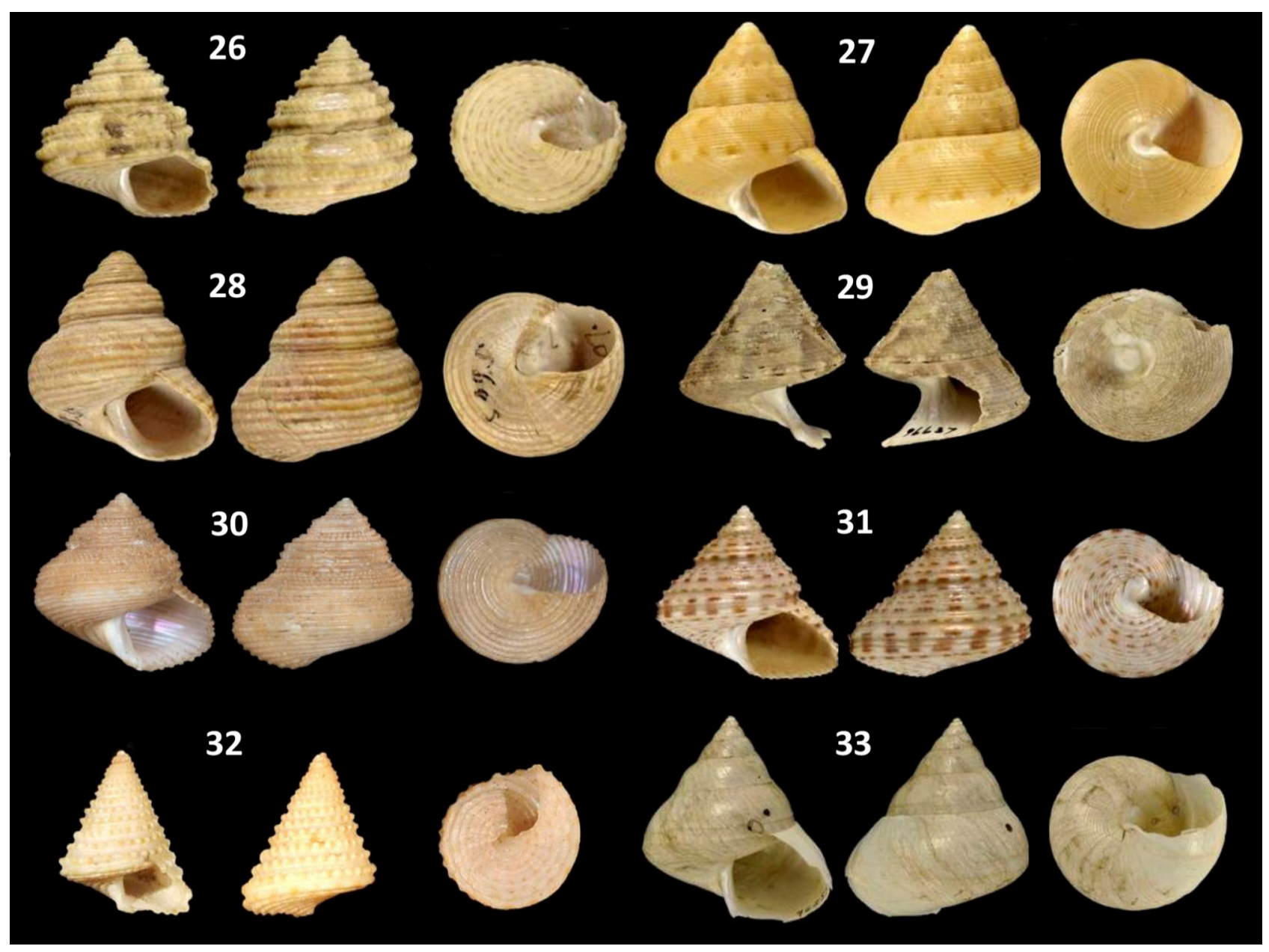

FIGURES 26-33. All type images provided by USNM. 26. Calliostoma formosum holotype (USNM 16261) $11.7 \mathrm{~mm}$, San Pedro, Los Angeles County, California. 27. Calliostoma gloriosum holotype (USNM 206134) 27.5 mm, Monterey Bay, Monterey County, California. 28. Trochus ligatus holotype (USNM 5608) $18.7 \mathrm{~mm}$, Puget Sound, Washington. 29. Calliostoma nepheloide syntype (USNM 96637) 25 mm, Panama Bay, Panama. 30. Calliostoma supragranosum holotype $14925,8.4 \mathrm{~mm}$, San Diego, San Diego County, California. 31. Calliostoma turbinum holotype (USNM 122578) $12 \mathrm{~mm}$, Santa Barbara Channel, Point Conception, Santa Barbara County, California, 183 m. 32. Calliostoma variegatum holotype (USNM 4201) ca. $6 \mathrm{~mm}$, Puget Sound, Washington. 33. Akoya platinum holotype (USNM 96558) $32 \mathrm{~mm}$, near Santa Barbara Island, Santa Barbara County, California, $756 \mathrm{~m}$.

\section{Acknowledgement}

Thanks to the following museums and individuals who made their collections available; SDMNH, LACM, SBMNH, SIO, CSDOMP, USNM, ANSP and CMNH, Dr. Ann McGowan-Tuskes for museum assistance and manuscript review; Dr. James McLean for his guidance over the years; Dr. Daniel Geiger for suggestions and manuscript critiques; Dr. Timothy Pearce, Paul Callomon, Lindsey Groves, Dr. Jann Vendetti and Paul Valentich-Scott for their museum assistance, and Dr. Christopher Meyer and Yolanda Villacampa (USNM) for photographs of eight holotypes in the USNM collection. 


\section{References}

Bouchet, P. \& Rocroi, J.P. (2005) Classification and nomenclator of gastropod families. Malacologia, 47, 1-397.

Carpenter, P.P. (1864a) Description of new marine shells from the coast of California Part 3. California Academy of Science Proceedings, 3, 207-224.

Carpenter, P.P. (1864b) Description of new marine shells from the coast of California Part 1. California Academy of Science Proceedings, 3, 155-159.

Carpenter, P.P. (1864c) Supplementary report on the present state of our knowledge with regard to the Mollusca of the west coast of North America. Reports of the British Association for the Advancement of Science, 33, 517-686.

Carpenter, P.P. (1865) Diagnoses Specierum et Varietatum novarum Moluscorum prope Sinum Pugetianum a Kennerlio Doctore, nuper decesso collectorum. Proceedings Academy Natural Sciences. Philadelphia, 17, 54-64.

Chemnitz, J.H. (1788) Neues Systematiches Conchylien-Cabinet, 10. Baur \& Raspe, Nürnberg. 376 pp. https://doi.org/10.5962/bhl.title.120067

Clark, R.N. (2018) New range data for five trochiform gastropods (Seguensiida [sic] \& Trochida) from Alaska. The Festivus, $50,114-116$.

Coan, E.V. \& Bogan, A.E. (1988) The Recent invertebrate taxa described by William More Gabb 1838-1878. Proceedings of the Academy of Natural Sciences, Philadelphia, 140, 273-284.

Dall, W.H. (1871) Descriptions of sixty new forms of mollusks from the west coast of North America and the North Pacific Ocean, with notes on others already described. American Journal of Conchology, 7, 93-160, pls. 13-16.

Dall, W.H. (1890) Scientific results of exploration by the USFC Steamer Albatross VII. Preliminary Report on the Collection of Mollusca and Brachiopods obtained in 1887-88. Proceedings of the United States National Museum, 12, 219-362, pls. 5-14.

Dall, W.H. (1896) Diagnoses of new species of mollusks from the west coast of America. Proceedings of the United States National Museum, 18, 7-20. https://doi.org/10.5479/si.00963801.1034

Dall, W.H. (1913) Diagnoses of new shells from the Pacific Ocean. Proceedings of the United States National Museum, 45, 587-597. https://doi.org/10.5479/si.00963801.45-2002.587

Dall, W.H. (1919) Description of new species of Mollusca from the North Pacific Ocean in the collection of the United States National Museum. Proceedings of the United States National Museum, 56, 293-371. https://doi.org/10.5479/si.00963801.56-2295.293

Dall, W.H. (1921) Summary of the marine shellbearing mollusks of the northwest coast of America, from San Diego, California, to the Polar Sea, mostly contained in the collection of the United States National Museum, with illustrations of hitherto unfigured species. United States National Museum, Bulletin, 112, 1-217, pls. 1-22.

Forbes, E. (1852) On the marine Mollusca discovered during the voyages of the Herald and Pandora. Proceedings of the Zoological Society London, for 1850, 270-274, p1s. 9-11.

Gabb, W. (1865) Description of new species of marine shells from the coast of California. Proceeding of the California Academy of Natural Sciences, 3, 182-190.

Gould, S. (1849) Descriptions of the following shells brought home by the U.S. Expedition. Proceedings of the Boston Society of Natural History, 3, 89-92.

Habe, T. (1961) Colored Illustrations of the Shells of Japan II. Hoikusha, Osaka, Japan. 182 pp., 66 pls.

Harbo, R.M. (2007) Shells \& Shellfish of the Pacific Northwest. Harbour Publishing, Madeira Park, BC, Canada. 271 pp.

Herrmannsen, A.N. (1846) Indicis Generum Malacozoorum Primordia, vol. 1. Theodor Fischer, Cassellis, 637 pp.

Hickman, C.S. \& McLean, J.H. (1990) Systematic revision and suprageneric classification of trochacean gastropods. Natural History Museum of Los Angeles County, Science Series, 35, 1-169.

Holyoak, A.R. (1988) Spawning and larval development of the trochid gastropod Calliostoma ligatum (Gould, 1849). The Veliger, 30, 369-371.

Houston, R.S. (1980) Mollusca. In: Brusca, R.C. (ed.). Common Intertidal Invertebrates of the Gulf of California. The University of Arizona Press, Tucson, Arizona, pp. 130-204.

Ikebe, N. (1942) Trochid Mollusca Calliostoma of Japan, fossil and Recent. Japanese Journal of Geology and Geography, 18, 249-282.

ICZN (1954) Direction 1. Opinions and Declarations Rendered by the International Commission on Zoological Nomenclature, $3,401-416$.

ICZN (1957) Opinion 456. Opinions and Declarations Rendered by the International Commission on Zoological Nomenclature, 15, 393-418.

Keen, A.M. (1975) On some West American species of Calliostoma. The Veliger, 17, 413-414.

LaGrange, J. (1992) Dredging off Nine Mile Bank, San Diego, California. The Festivus, 24, 47-58. 
LaGrange, J. (1998) California's deep water calliostomas. The Festivus, 30, 45-47.

Lightfoot, J. (1786) A Catalogue of the Portland Museum, Lately the Property of The Duchess Dowager of Portland Deceased: Which will be Sold by Auction. Skinner, Portland. 20 pp.

Martyn, T. (1784) The Universal Conchologist. [privately published], London. 39 pp., 80 pls.

McLean, J.H. (1969) Marine shell of Southern California. Natural History Museum of Los Angeles County Science Series, 24, $1-104$.

McLean, J.H. (1970) Notes on the deep water calliostomas of the Panamic province, with descriptions of six new species. The Veliger, 12, 421-426.

McLean, J.H. (1971) Archaeogastropoda. In: Keen, A.M. (Ed.), Sea Shells of the Tropical West America. Marine Mollusks from Baja California to Peru, Second Edition. Stanford University Press, Stanford, pp. 307-363.

McLean, J.H. (1984) New species of Northeast Pacific archaeogastropods. The Veliger, 26, 233-239.

McLean, J.H. (2012) Detrital feeding in Xeniostoma inexpectans, new genus, new species, and new subfamily Xeniostomatinae of Calliostomatidae (Gastropoda:Vetigastropoda), hosted by hexactinellid sponges of the Aleutian Islands, Alaska. The Nautilus, 126, 89-97.

Menke, K.T. (1850) Conchylien von Mazatlan, mit kritischen Anmerkungen. Zeitschrift für Malakozoologie, 7, $161-176$.

Middendorff, T.A. von. (1849) Beiträge zu einer Malacozoologica Rossica, II. Aufzählung und Beschreibung der zur Meeresfauna Russlands gehörigen Einschaler. Mémoires de l'Académie Impériale des Sciences de St-Petersbourg, Sixième Série, Sciences Naturelles, 6, 329-516, pls. 1-11.

Morris, R.H., Abbott, D.T. \& Haderlie, E.C. (1990) Intertidal Invertebrates of California. Stanford University Press, Stanford. 658 pp., 200 pls.

Oldroyd, I.S. (1927) The Marine Shells of the West Coast of North America, 3. Stanford University Press, Stanford. 339 pp, pls. $72-108$.

Otsuka, Y. (1939) A new Calliostoma from Japan. Venus, 9, 27-29.

Palmer, K.V.W. (1958) Type specimens of marine Mollusca described by P.P. Carpenter from the west coast (San Diego to British Columbia). The Geological Society of America Memoir, 76, 1-376. https://doi.org/10.1130/MEM76-p1

Perron, F. (1975) Carnivorous Calliostoma (Prosobranchia: Trochidae) from the Northeastern Pacific. The Veliger, 18, $52-54$.

Philippi, R.A. (1851) Die Kreiselsschecken Order Trochoideen (Gattungen Turbo, Trochus, Solarium, Rotella, Delphinula, Phasianella.) in: Küster, H.C. (ed.) Systematisches Conchylien Cabinet von Martin und Chemnitz 2(3), Bauer und Raspe, Nürnberg, pp. 1-372, pls. 1-49.

Reeve, L. (1842a) Descriptions of new species of shells belonging to the genera Trochus and Turbo. Proceedings of the Zoological Society London, 10, 184-186.

Reeve, L. (1842b) Conchologia Systematica, vol. 2. Longman, Brown, Green, and Longmans, London. 337 pp., 167 pls.

Sasaki, T. (2000) Family Trochidae. In: Okutani, T. (ed.) Marine Mollusks in Japan, Tokai University Press, Tokyo. Pp. $55-83$.

Severns, M. (Ed.) (2011) Shells of the Hawaiian Islands. The Sea Shells. ConchBooks, Hackenheim. 563 pp.

Skoglund, C. \& Koch, R. (1993) New distributional information for Panamic province Archaeogastropoda (Mollusca). The Festivus, 35, 116-118.

Smith, A.G. \& Gordon, M. (1948) The marine mollusks and brachiopods of Monterey Bay, California, and Vicinity. Proceedings of the California Academy of Science, $4^{\text {th }}$ series, 26, 228-229, pls. 3-4.

Stone, R.P., Masuda, M.M., \& Karinen, J.F. (2014) Assessing the ecological importance of red tree coral thickets in the eastern Gulf of Alaska. ICES Journal of Marine Sciences, 72, 900-915. https://doi.org/10.1093/icesjms/fsu190

Swainson, W. (1840) A Treatise on Malacology or Shells and Shell-fish. Longman \& Taylor, London. 418 pp.

Thiele, J. (1924) Revision des Systems der Trochacea. Mitteilungen aus dem Zoologischen Museum in Berlin, 11, 47-74, 1 pl.

Tuskes, P.M. \& Clark, R. (2018) A new species of Otukaia (Calliostomatidae) from Alaska. The Festivus, 50, $236-238$.

Tuskes, P.M. \& Tuskes, A.M. (2019) Calliostoma and Akoya of the Californian Marine Province (Gastropoda: Calliostomatidae). The Festivus, 35, 11-28.

Valenciennes, A. (1846) Mollusques. In Du-Petit-Thouars, M.A. (ed.), Voyage Autour du Monde sur la Frégate La Venus Pendant les Années 1836-1839. Atlas de Zoologie, pls. Mollusques 1-24.

Williams, S.T., Donald, K.M., Spencer, H.G. \& Nakano, T. (2010) Molecular systematics of the marine gastropod families Trochidae and Callostomatidae (Mollusca superfamily Trochiodea). Molecular Phylogenetics and Evolution, 54, 783-809. https://doi.org/10.1016/j.ympev.2009.11.008

Wood, W. (1828) Supplement to the Index Testaceologicus; or a Catalogue of Shells, British and Foreign [Privately Published] London. 58 pp., 8 pls. 